\title{
IDENTIFICACIÓN DE PROPIEDADES DINÁMICAS DE UN MODELO ESTRUCTURAL SOMETIDO A VIBRACIÓN AMBIENTAL Y VIBRACIÓN FORZADA EMPLEANDO MESA VIBRADORA
}

\author{
Henao Ángel D. ${ }^{(1)}$, Botero Palacio J.C. ${ }^{(2)}$, Murià Vila D..$^{(3)}$
}

\begin{abstract}
RESUMEN
Con el objeto de validar los resultados obtenidos con los nuevos equipos de instrumentación sísmica adquiridos por la Universidad EAFIT e implementar una metodología de análisis para la identificación de las propiedades dinámicas de estructuras, se construyó un modelo de una estructura de acero de cinco niveles y se elaboró un modelo numérico. La estructura fue sometida a pruebas de vibración ambiental y de vibración forzada empleando una mesa vibradora. Las frecuencias de vibración, formas modales y fracciones de amortiguamiento crítico se estimaron aplicando un análisis espectral. Los resultados obtenidos en las pruebas son coherentes y consistentes con los resultados numéricos. Esto permite validar la metodología empleada y determinar el buen desempeño de los equipos adquiridos.
\end{abstract}

Palabras Clave: Vibración Ambiental; vibración forzada; procesos estocásticos; identificación experimental de sistemas

\section{IDENTIFICATION OF DYNAMIC PROPERTIES OF A STRUCTURAL MODEL SUBJECTED TO AMBIENT VIBRATION AND FORCED VIBRATION USING SHAKING TABLE}

\begin{abstract}
In order to validate the results obtained by the recently acquired seismic monitoring equipment at Universidad EAFIT, and to implement a numerical framework for the identification of the dynamic properties of a structure, a five-storey steel building was built and its numerical model was developed. The scaled model was subjected to ambient, forced vibration and shaking table test. The modal frequencies, vibration modes and damping ratios were estimated using a spectral analysis approach. Experimental results are in good agreement with numerical estimates. This validates the methodology used and the good performance of the monitoring equipment acquired.
\end{abstract}

Keywords: Ambient vibration, forced vibration, stochastic processes, experimental system identification

Artículo recibido el 12 de agosto de 2013 y aprobado para su publicación el 17 de mayo de 2014. Se aceptarán comentarios y/o discusiones hasta cinco meses después de su publicación.

(1) Universidad EAFIT, Cra. 49 \# 7 sur 50, Medellín-Colombia, dhenaoa1 @ eafit.edu.co

${ }^{(2)}$ Universidad EAFIT, Cra. 49 \# 7 sur 50, Medellín-Colombia, jcbotero@eafit.edu.co

${ }^{(3)}$ Instituto de Ingeniería, Universidad Nacional Autónoma de México, Ciudad Universitaria, 04510, México D.F., dmv@pumas.ii.unam.mx 


\section{INTRODUCCIÓN}

La ingeniería estructural, en su continuo desarrollo, ha implementado técnicas experimentales que buscan una adecuada comprensión de lo que ocurre con las estructuras en la etapa posterior a la construcción y que permitan la identificación de las propiedades dinámicas reales de las mismas durante su vida útil. La instrumentación de edificaciones surge como una posibilidad de tener acceso a ese tipo de información que permite comprender las propiedades dinámicas de la estructura, evaluar el diseño de la edificación una vez construida o comprender e identificar posibles daños después de la ocurrencia de un evento sísmico.

Se identifican tres tipos de mediciones que se pueden realizar en estructuras instrumentadas: mediciones sísmicas, de vibración ambiental y de vibración forzada. Las mediciones sísmicas son las que se realizan a partir de eventos sísmicos reales ocurridos, que afectan directamente a una edificación que cuenta con instrumentación sísmica permanente. Por su parte, las pruebas de vibración ambiental consisten en medir las vibraciones de las estructuras producidas por excitaciones de carácter ambiental, como los son las producidas por el tránsito de vehículos y el viento (Murià Vila y Gonzalez Alcorta, 1995), sin embargo las amplitudes del movimiento estructural en el caso de vibraciones ambientales dependen principalmente de la velocidad del viento, ya que el viento es usualmente una fuente de excitación más efectiva que los microtemblores o fuentes dentro de la estructura (Trifunac, 1972). Finalmente, las vibraciones forzadas son aquellas en las que se genera una excitación de manera artificial, de tal modo que afecte la estructura a la cual se le desean realizar las mediciones.

A la par con la instrumentación de edificaciones y técnicas de medición se deben desarrollar herramientas computacionales y numéricas que posibiliten el análisis de los registros obtenidos mediante las teorías matemáticas y estadísticas existentes. El tipo de análisis requerido está directamente ligado con el tipo de excitación que experimenta la estructura, la magnitud de las aceleraciones y su duración.

La teoría de los procesos estocásticos y el análisis espectral son los instrumentos utilizados para el análisis no paramétrico de este tipo de señales en estructuras instrumentadas sometidas a vibraciones ambientales, con la ayuda de éstos es posible la identificación de las propiedades dinámicas de una edificación (Bendat y Piersol, 2010). Por su parte para excitaciones de mayor magnitud pero de duraciones significativamente menores se utilizan los cocientes espectrales.

La investigación realizada busca validar los resultados obtenidos por los equipos de instrumentación sísmica adquiridos por la Universidad EAFIT para fines de investigación y docencia, e implementar una metodología de análisis espectral con el fin de evaluar las propiedades dinámicas de una estructura mediante la realización de pruebas de vibración ambiental y vibración forzada.

Para validar los resultados obtenidos con los equipos de instrumentación se construyó un modelo estructural de acero, en donde se calcularon sus propiedades dinámicas a partir de modelos numéricos considerando sus características reales. Adicionalmente, se identificaron las propiedades dinámicas a partir de un análisis espectral, empleando los resultados obtenidos y las herramientas de cómputo desarrolladas en el proyecto. Los resultados obtenidos mediante ambos procedimientos resultaron ser coherentes entre sí. 


\section{PROGRAMA EXPERIMENTAL}

Para el desarrollo de la investigación se diseñó y construyó un modelo estructural de acero, el cual fue instrumentado. El modelo se sometió a diferentes niveles de excitación y se llevaron a cabo varios tipos de pruebas con él.

\section{Modelo de análisis}

El modelo utilizado consta de cinco niveles de planta cuadrada, con cuatro columnas rectangulares de perfiles tubulares en los extremos, unidas por vigas igualmente rectangulares y con placas de acero que simulan el sistema de entrepiso y que actúan como las masas principales del sistema, proporcionándole la condición de diafragma rígido. Todas las conexiones entre trabes y columnas son atornilladas. Las dimensiones de la estructura se seleccionaron de tal forma que el periodo fundamental fuera cercano a 0.1 s por nivel ( $0.5 \mathrm{~s})$. La Figura 1 muestra el modelo utilizado para el análisis.

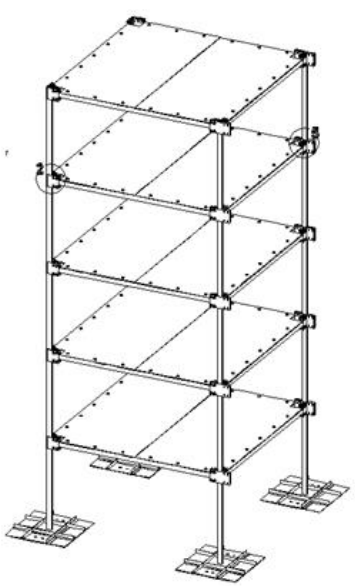

a. Modelo Proyectado

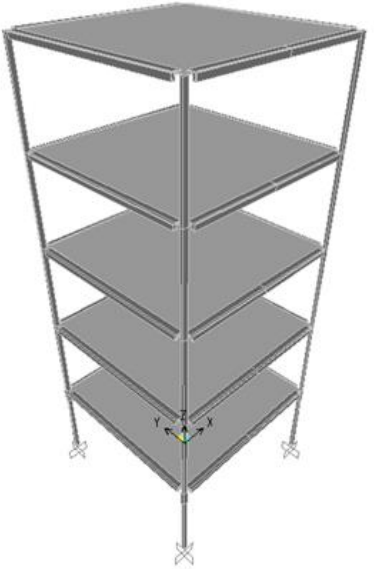

b. Modelo Analítico

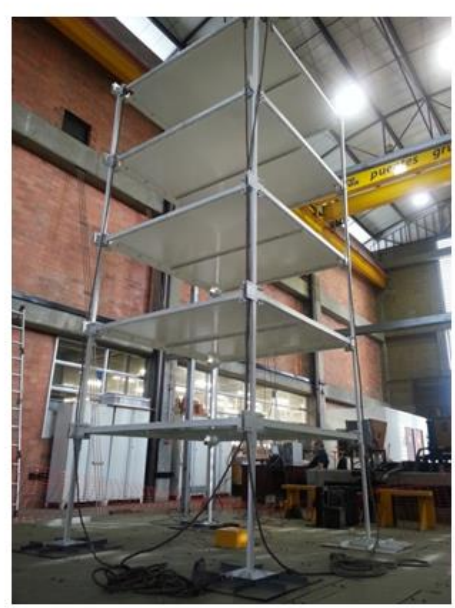

c. Modelo Construido

Figura 1. Características de la estructura de análisis

Con el modelo estructural de análisis se buscó eliminar al máximo los factores externos que pudieran impactar los resultados del modelo matemático (teóricos). Las dimensiones y características principales del modelo se muestran en la Tabla 1; los pesos estipulados fueron suministrados por la compañía constructora.

La selección de las direcciones ortogonales para el análisis y para la excitación del modelo se realizó de tal forma que éstas fueran paralelas a las caras de las columnas. La dirección X coincide con el eje de mayor inercia de las columnas y la dirección Y con el de menor inercia. De acuerdo con estas direcciones se dispusieron los sensores para la toma de los datos. 
Tabla 1. Características del modelo de análisis

\section{Modelo de Análisis}

\begin{tabular}{lc}
\hline Sección columnas rectangulares $(\mathrm{mm})$ & $50 \times 30 \times 1.5$ \\
Sección vigas rectangulares $(\mathrm{mm})$ & $50 \times 30 \times 1.5$ \\
Número de niveles & 5 \\
Dimensiones generales $(\mathrm{m})$ & $2.0 \times 2.0 \times 5.0$ \\
Altura de entrepiso $(\mathrm{m})$ & 1 \\
Peso específico acero $\left(\mathrm{kN} / \mathrm{m}^{3}\right)$ & 76.98 \\
Placas de piso $(\mathrm{m})$ & $2.0 \times 2.0 \times 0.006$ \\
Peso placa entrepiso $(\mathrm{kN})$ & 1.9 \\
Peso del modelo. Incluye cimentación $(\mathrm{kN})$ & 13.7 \\
\hline
\end{tabular}

Tabla 2. Períodos y frecuencias de vibración obtenidos del análisis modal

\begin{tabular}{ccc}
\hline Modo & Período $($ s) & Frecuencia $(\mathrm{Hz})$ \\
\hline $\mathrm{Tx}_{1}$ & 0.51 & 1.95 \\
$\mathrm{Ty}_{1}$ & 0.66 & 1.51 \\
$\mathrm{Tz}_{1}$ & 0.37 & 2.67 \\
$\mathrm{Tx}_{2}$ & 0.17 & 6.03 \\
$\mathrm{Ty}_{2}$ & 0.22 & 4.49 \\
$\mathrm{Tz}_{2}$ & 0.12 & 8.09 \\
$\mathrm{Tx}_{3}$ & 0.10 & 10.46 \\
$\mathrm{Ty}_{3}$ & 0.14 & 7.31 \\
$\mathrm{Tz}_{3}$ & 0.07 & 13.60 \\
\hline
\end{tabular}

El diseño y análisis estructural del modelo se llevó a cabo mediante el empleo del programa de cómputo SAP2000TM (Computers and Structures Inc., 2012). El esquema de la modelación realizada se puede observar en la Figura 1, y en la Tabla 2 se muestran los períodos y las frecuencias de vibración obtenidos del análisis modal teórico. El modelo numérico se ajustó a las condiciones reales de construcción (secciones utilizadas, conexiones, pesos de los elementos, etc.) con el fin de que representara más apropiadamente las condiciones reales del modelo construido y así obtener una adecuada correlación entre los resultados teóricos y experimentales.

\section{Equipos utilizados en las pruebas}

Para el registro de las señales de los experimentos se utilizaron acelerómetros triaxiales de fabricación neozelandesa denominados CUSP-Me y desarrollados por Canterbury Seismic Instruments. El equipo está compuesto por una unidad central de 16 canales a la cual se conectan acelerómetros de alta precisión. La Figura 2 muestra los aparatos utilizados en la medición. 


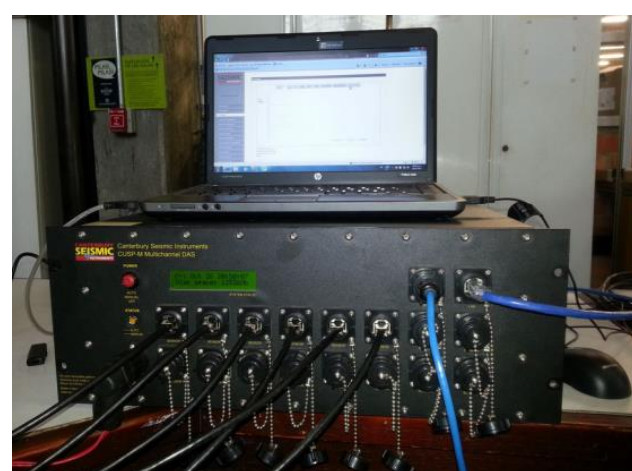

a) Unidad central de control

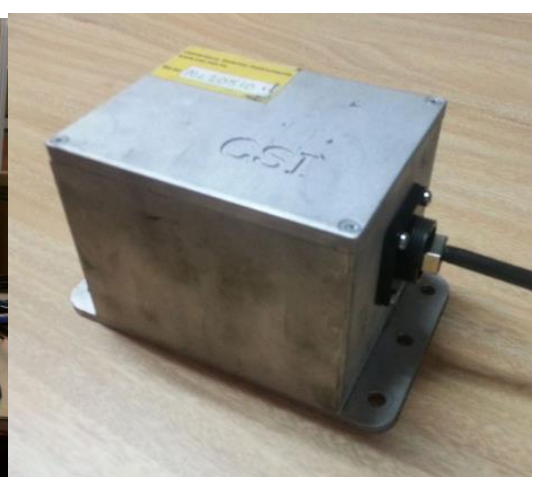

b) Acelerómetro triaxial

Figura 2. Equipos de instrumentación adquiridos y utilizados en los ensayos

El CUSP-Me es un registrador para monitoreo dinámico multicanal al cual se le pueden conectar hasta 16 sensores triaxiales, para un total de 48 registros simultáneos. La transmisión de la información entre los sensores y la unidad central se lleva a cabo mediante cables convencionales de Ethernet, dado que el sensor digitaliza los datos. Esto representa una ventaja sobre otro tipo de instrumentación con respecto a la movilidad de los equipos ya que es posible la ubicación de los sensores a distancias de hasta $100 \mathrm{~m}$ aproximadamente de la unidad central sin el requerimiento de amplificadores de señal ni el uso de cables blindados. La unidad central se conecta a una computadora PC con un procesador CORE i5 a través del cual se definen los parámetros de control.

Los acelerómetros conectados al registrador CUSP-Me, registran datos con una frecuencia de muestreo de 200 muestras por segundo y con una precisión de $\pm 0.001 \mathrm{Gal}\left(1 \mathrm{Gal}=1 \mathrm{~cm} / \mathrm{s}^{2}\right)$, es decir 0.0001 por ciento del valor de la gravedad y pueden registrar eventos con aceleraciones inferiores a cuatro veces el valor de la gravedad $( \pm 4 \mathrm{~g})$.

Para las pruebas de vibración forzada se utilizó la mesa vibradora del laboratorio de investigación en ingeniería sísmica de la Universidad EAFIT. La mesa vibradora es una plataforma metálica de $36 \mathrm{~m}^{2}$, con capacidad de carga vertical de $70 \mathrm{t}$, altura libre de $9.5 \mathrm{~m}$, velocidad máxima de $80 \mathrm{~cm} / \mathrm{s} \mathrm{y}$ desplazamiento máximo de $10 \mathrm{~cm}$, todo controlado por un sistema electrónico de alta precisión.

\section{Descripción de las pruebas realizadas}

En el modelo construido se llevaron a cabo pruebas de vibración ambiental y de vibración forzada con excitación en la base para determinar las propiedades dinámicas de traslación y de torsión.

Con el fin de poder disponer de los datos necesarios para visualizar la respuesta dinámica tridimensional de la estructura, y teniendo en cuenta el número de sensores, se diseñaron dos arreglos de instrumentación diferentes para cada análisis. La instrumentación de la estructura para ambos ensayos se muestra en la Figura 3. Ambos tipos de análisis se llevaron a cabo tanto para los ensayos de vibración ambiental como para los de vibración forzada. Con el análisis de los registros del arreglo A se pretenden obtener las propiedades de traslación horizontal y con el arreglo B las de los modos de torsión. 

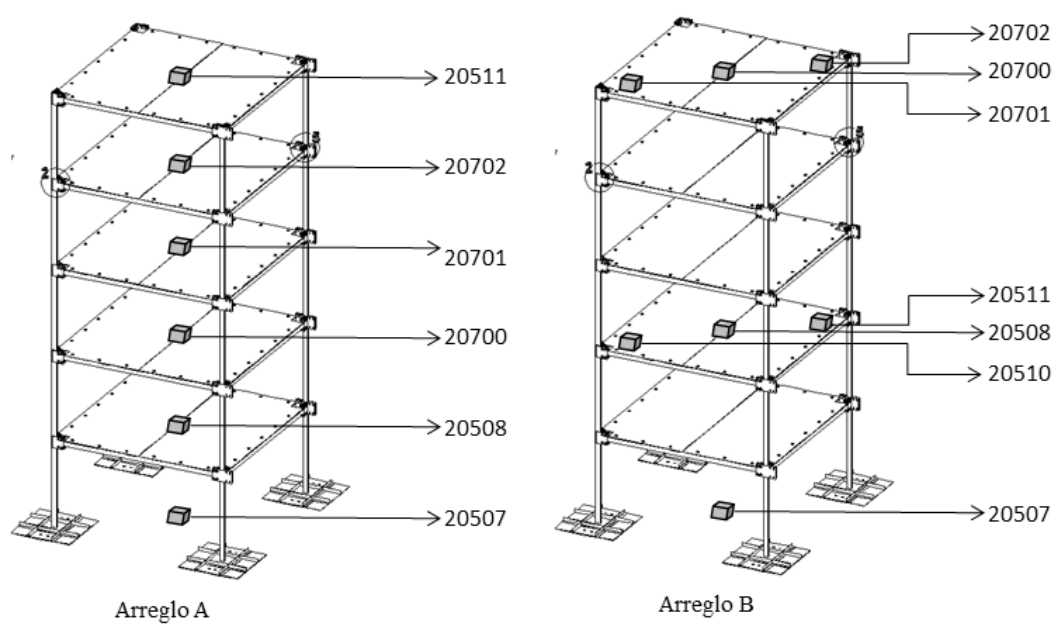

Figura 3. Disposición de los dos arreglos de acelerómetros triaxiales empleados en las pruebas

Para la identificación de las pruebas de traslación, con el arreglo A (Figura 3), se dispusieron sensores en cada uno de los niveles ubicados el mismo eje vertical. El sensor de la base registra la señal de entrada y se puede evaluar la respuesta en cada nivel de la estructura. Con este arreglo de instrumentos es posible identificar frecuencias de traslación, formas modales y amortiguamiento de la estructura.

En las pruebas de torsión, con el arreglo B (Figura 3), se dispuso de tres sensores en dos de los niveles y un sensor más en la base; en los niveles instrumentados se cuenta con un sensor en el centro y los otros dos en esquinas opuestas, esto con el fin de identificar los movimientos rotacionales alrededor del eje vertical de la estructura propios de los modos de torsión.

Por su parte para las pruebas de vibración ambiental se instalaron los equipos en el modelo y se tomaron registros de treinta minutos de duración, con una frecuencia de muestreo de $200 \mathrm{~Hz}$. La finalidad de la duración de estos registros es reducir al máximo los errores estadísticos adscritos a los estimativos que utiliza la metodología de análisis (Bendat y Piersol, 1993).

Para las pruebas de vibración forzada se empleó la mesa vibradora del laboratorio de ingeniería sísmica de la Universidad EAFIT. Debido a que el mecanismo de activación de la mesa funciona mediante la aplicación de desplazamientos en el gato hidráulico, los cuales generan el movimiento y consiguiente aceleración de la mesa, se proporcionaron varias magnitudes de desplazamientos que a su vez produjeron aceleraciones máximas en la mesa entre 25 y 75 Gal. Estas magnitudes de aceleración se seleccionaron con la finalidad de que el modelo estructural se mantuviera en el rango elástico, propio de las vibraciones ambientales, y que no se indujeran no linealidades al sistema que además de ser difíciles de analizar dañarían de manera permanente la estructura. La duración de la excitación inducida fue de $30 \mathrm{~s}$ y las pruebas se realizaron para una de las direcciones principales (Dirección X). El modelo no presentó falla ante las aceleraciones provocadas. Se presentan los resultados de la prueba de mayor magnitud realizada. 


\section{ANÁLISIS DE RESULTADOS}

Para el análisis de las pruebas de vibración ambiental se utiliza la teoría de procesos estocásticos y datos aleatorios (Bendat y Piersol, 2010). Su implementación permite la obtención de espectros de potencia, cocientes espectrales y funciones de coherencia para identificar las frecuencias, amortiguamientos y formas modales.

Dichas funciones, denominadas de densidad espectral son la representación en el dominio de la frecuencia de las funciones de correlación y proveen básicamente la misma información, con la diferencia que históricamente las funciones de correlación son de mayor utilidad matemática y estadística, mientras que las funciones de densidad espectral se han desarrollado más como una herramienta ingenieril (Bendat y Piersol, 1993). Este desarrollo estadístico, también se denomina técnicas no paramétricas y consiste en determinar las características del sistema estructural a partir del análisis de los datos en los dominios del tiempo y de la frecuencia (Murià Vila, 2007).

La fracción de amortiguamiento crítico para cada modo de vibración se obtiene mediante el empleo de la metodología del ancho de banda del espectro de potencia (Kawasumi y Shima, 1965), el cual puede ser corregido empleando la corrección por sobrestimación del amortiguamiento (Boroschek y Hernandez, 2010).

Por su parte, para las pruebas de vibración forzada se utilizaron las funciones de transferencia del sistema, las cuales están definidas como el cociente entre los espectros de potencia de dos señales registradas.

\section{Pruebas de vibración ambiental para identificar propiedades de traslación}

La Figura 4 muestra los registros típicos de vibración ambiental obtenidos en la base y en la azotea del modelo estructural, para las dos direcciones de análisis.

Para la identificación de las frecuencias de traslación se llevaron a cabo dos tipos de comparaciones entre los registros de los niveles instrumentados: una consistente en confrontar un nivel dentro de la estructura (preferiblemente la azotea) con uno fuera de ella o en la base, y la otra cotejando registros de dos niveles internos de la edificación. Al comparar dos puntos instrumentados, uno al interior y otro fuera de la estructura se analiza la información contenida especialmente en los espectros de potencia y en los cocientes espectrales.

La Figura 5 muestra los espectros de potencia de los puntos analizados, éstos dan información de cada punto de manera independiente. El espectro de potencia de la base contiene esencialmente información del contenido frecuencial del sitio, mientras que el de la azotea muestra picos tanto en las frecuencias del sistema como las del sitio. 

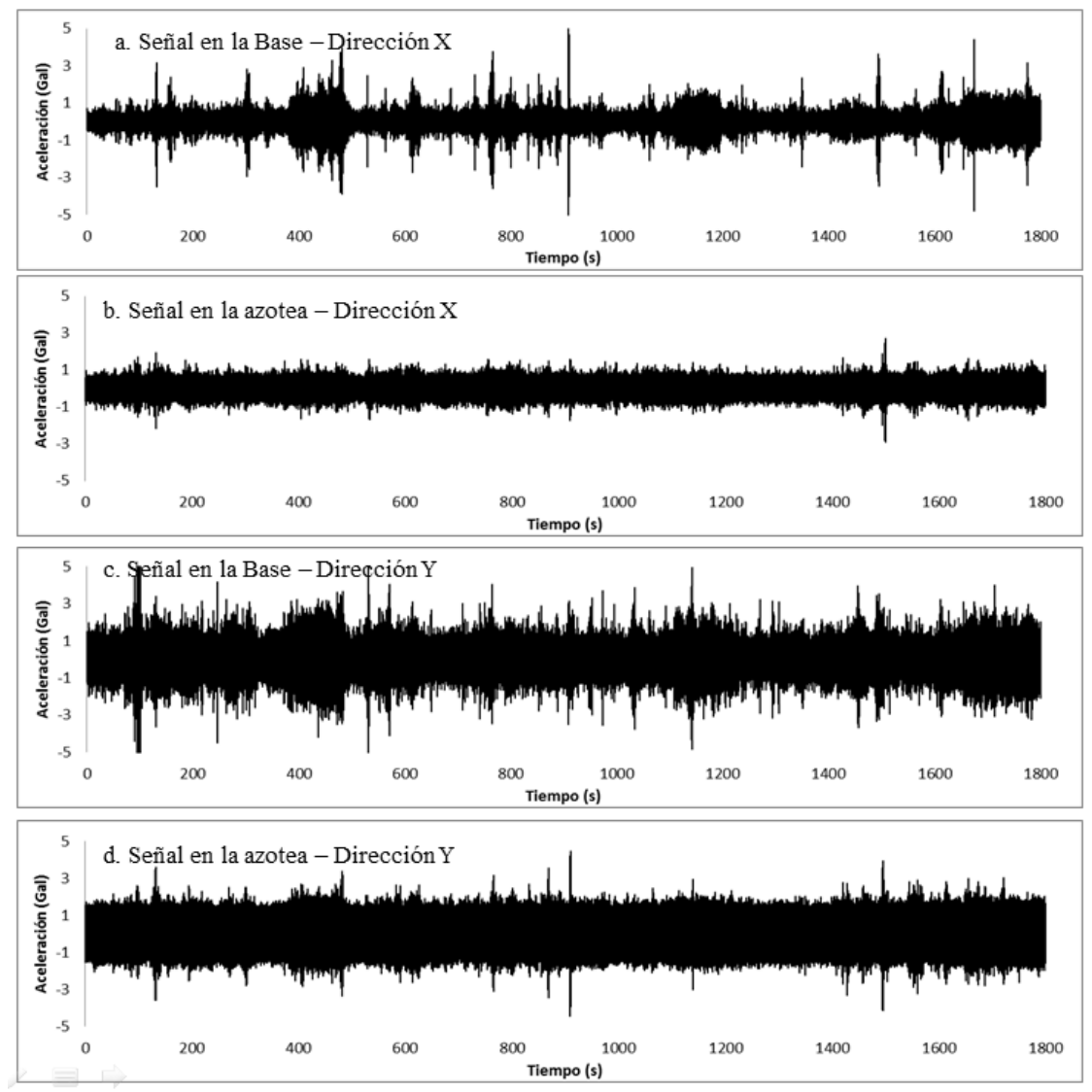

Figura 4. Señales en el tiempo obtenidas para las pruebas de vibración ambiental en traslación
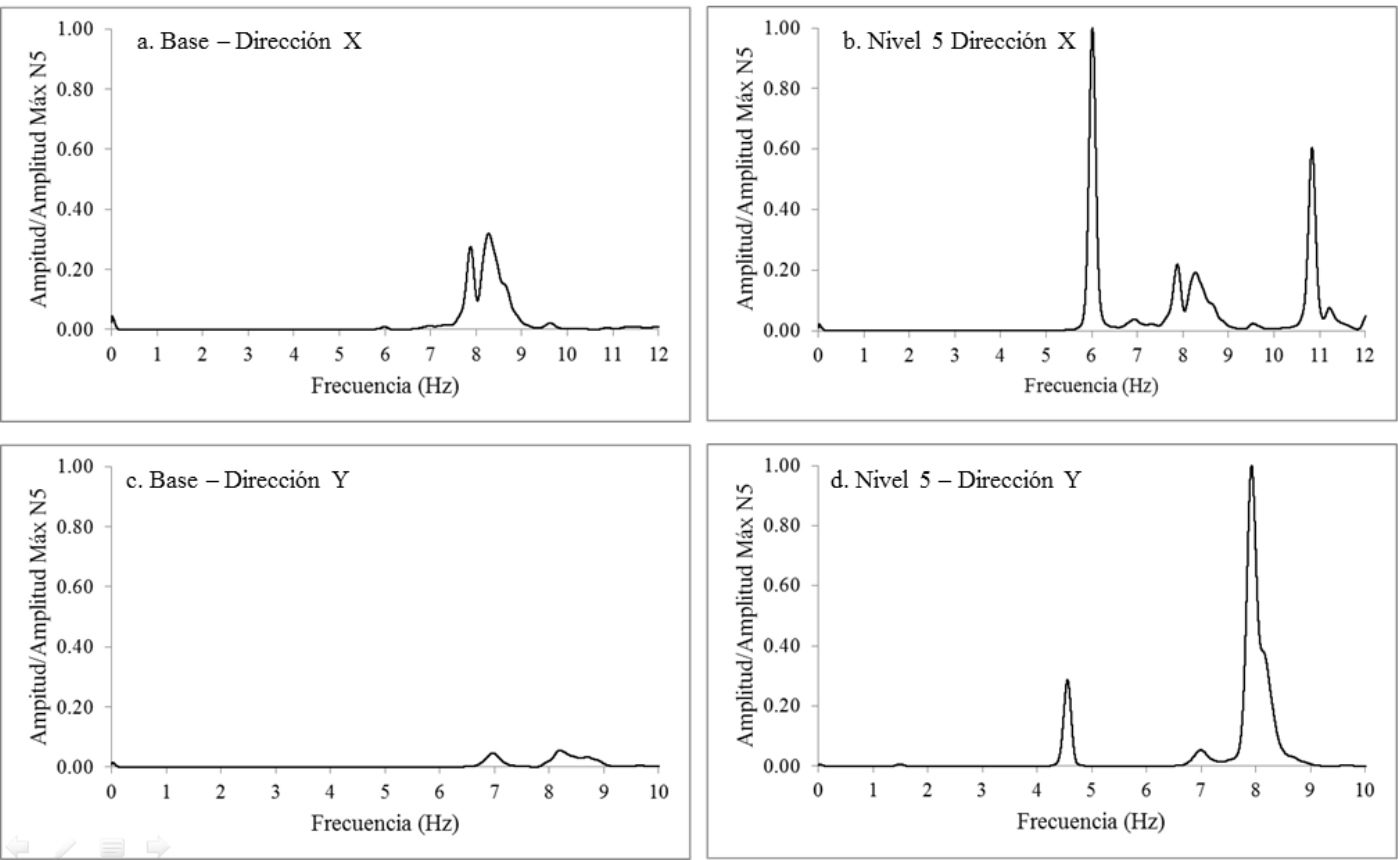

Figura 5. Espectros de potencia obtenidos a partir de los registros de los sensores ubicados en la base y en el nivel N5 (azotea) para las pruebas de vibración ambiental en translación 
La Figura 6 muestra el cociente espectral entre los espectros de potencia de estas dos señales. Éste muestra picos asociados a las frecuencias del sistema en la dirección de análisis, y suprimen el contenido frecuencial del terreno. Estos picos están bien definidos y al compararlos con la información obtenida de los espectros independientes provee la información acerca de las frecuencias del sistema. Se identifican como las frecuencias de vibración de la estructura 1.9, 6.1 y $10.8 \mathrm{~Hz}$ para los tres primeros modos en la dirección X y 1.5, 4.6 y 7.9 Hz para los tres primeros modos en la dirección Y.
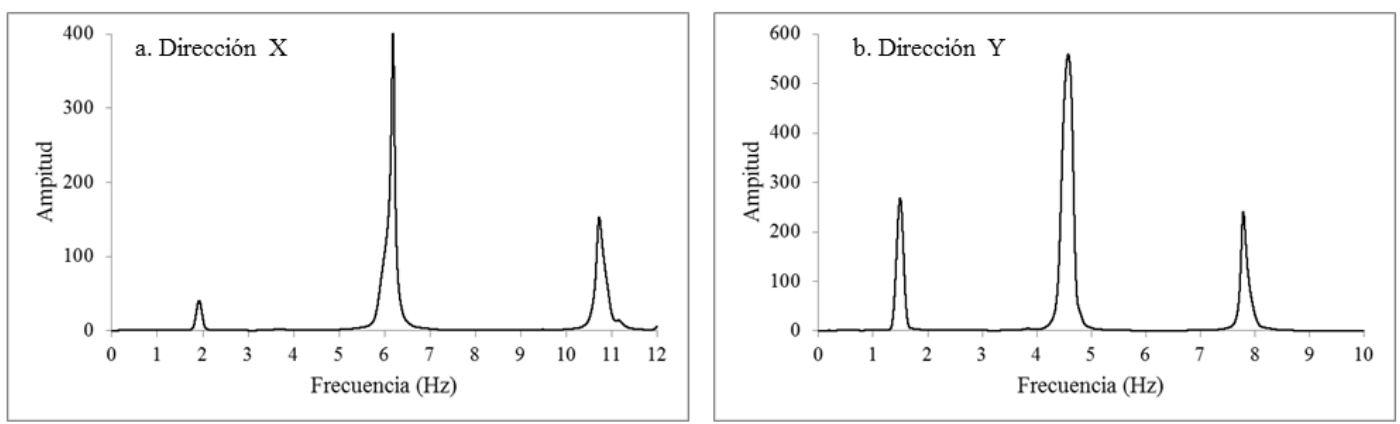

Figura 6. Cociente espectral entre los espectros de potencia obtenidos a partir de los registros de los sensores ubicados en el nivel N5 (azotea) y en la base para las pruebas de vibración ambiental en traslación

Complementando el análisis anterior se realiza la comparación entre dos puntos instrumentados dentro de la estructura y se analiza la información contenida en los espectros de potencia y funciones de coherencia. Es posible realizar tantas comparaciones como niveles instrumentados se tengan y los resultados deben ser consistentes. Para dicho análisis se seleccionan los resultados de los niveles dos y cinco del modelo instrumentado, cuyos espectros de potencia se muestran en la Figura 7.

La función de coherencia por su parte, presenta valores altos, es decir cercanos a uno, para las frecuencias de la estructura (Figura 8). Si bien la coherencia es alta en los puntos de interés, también lo es para otros rangos diferentes, por lo que esta información por sí sola no es suficiente para el análisis de las propiedades de la estructura. Sin embargo, las funciones de coherencia entre los registros de los sensores ubicados en los puntos intermedios de la estructura, permiten realizar una verificación de los resultados calculados y de esta manera confirmar los valores de frecuencias modales identificadas mediante los espectros de potencia y cocientes espectrales en las comparaciones descritas. 

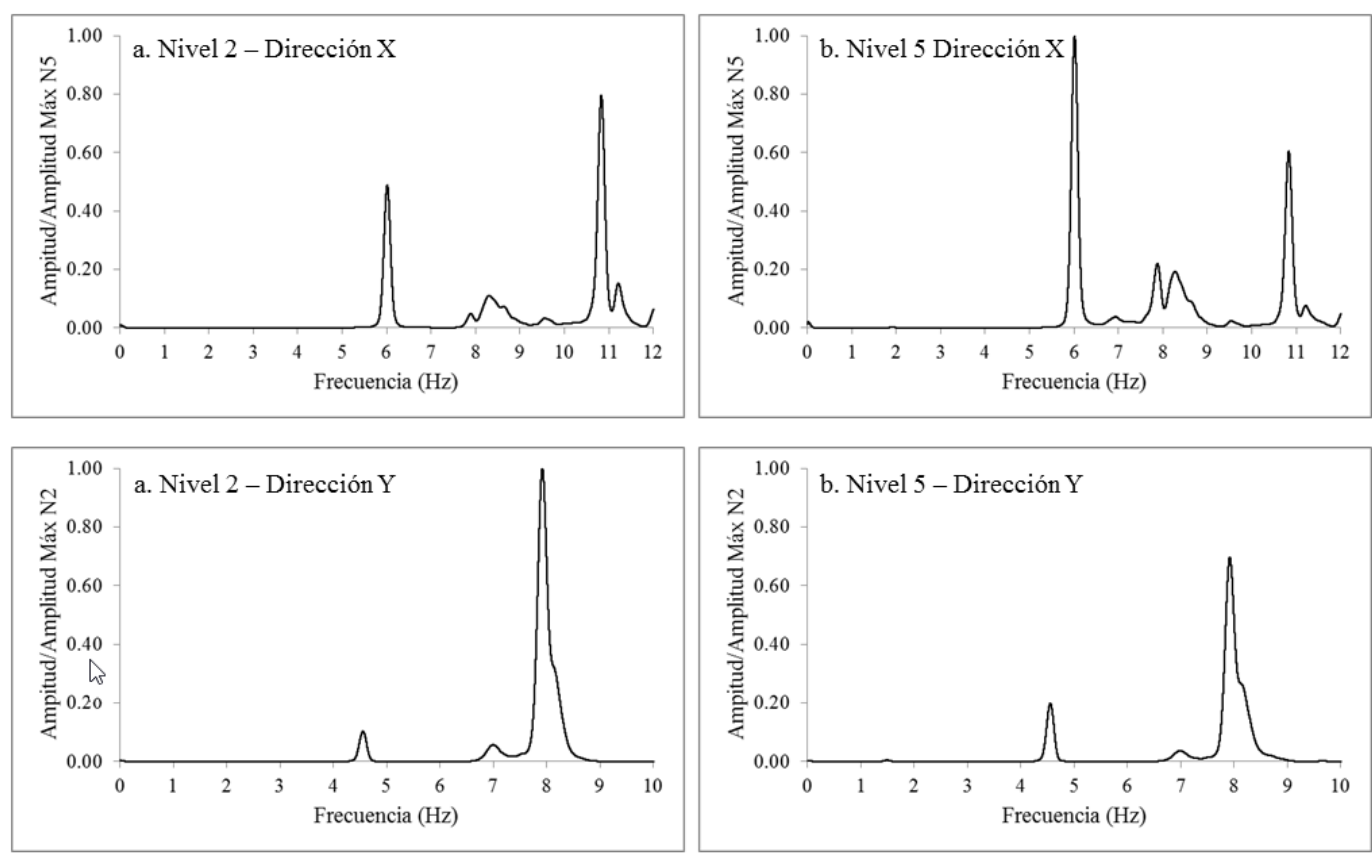

Figura 7. Espectros de potencia obtenidos a partir de los registros de los sensores ubicados en los niveles N2 y N5 (azotea) para las pruebas de vibración ambiental en traslación
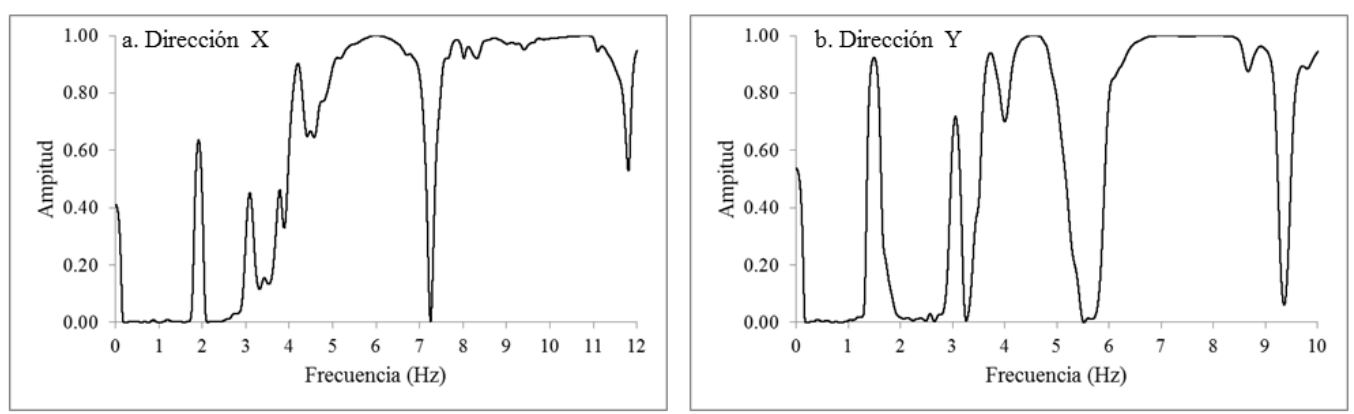

Figura 8. Función de coherencia obtenida a partir de los registros de los sensores ubicados en los niveles N2 y N5 (azotea) para las pruebas de vibración ambiental en traslación

\section{Evaluación de formas modales a partir de pruebas de vibración ambiental}

Otra de las propiedades que es posible identificar con este tipo de pruebas, son las formas modales de vibración de la estructura. A partir de los espectros de potencia de todos los niveles instrumentados se pueden identificar dichas formas, basándose en la amplitud que alcanza el espectro de potencia para cada nivel en cada frecuencia de vibración.

La metodología consiste en conocer la amplitud que alcanza la estructura en cada nivel a partir del espectro de potencia, y conocer del espectro de fase del análisis cruzado de las señales, la dirección en la que los niveles se encuentran relativamente uno del otro. 
La Figura 9 ilustra los modos de vibración encontrados para las dos direcciones ortogonales de análisis. Para cada frecuencia identificada como un modo de vibración de la estructura, se normalizan con respecto al nivel con mayor y se leen los máximos de cada uno. Conjuntamente se identifica la fase entre pares de niveles; si los niveles se encuentran en fase (ángulo de fase igual a 0), las amplitudes ocurren con el mismo signo, si se encuentran en fase opuesta (ángulo de fase igual a $\pm \pi$ ), las amplitudes se escriben con signo contrario. De esta manera es posible construir las formas modales.
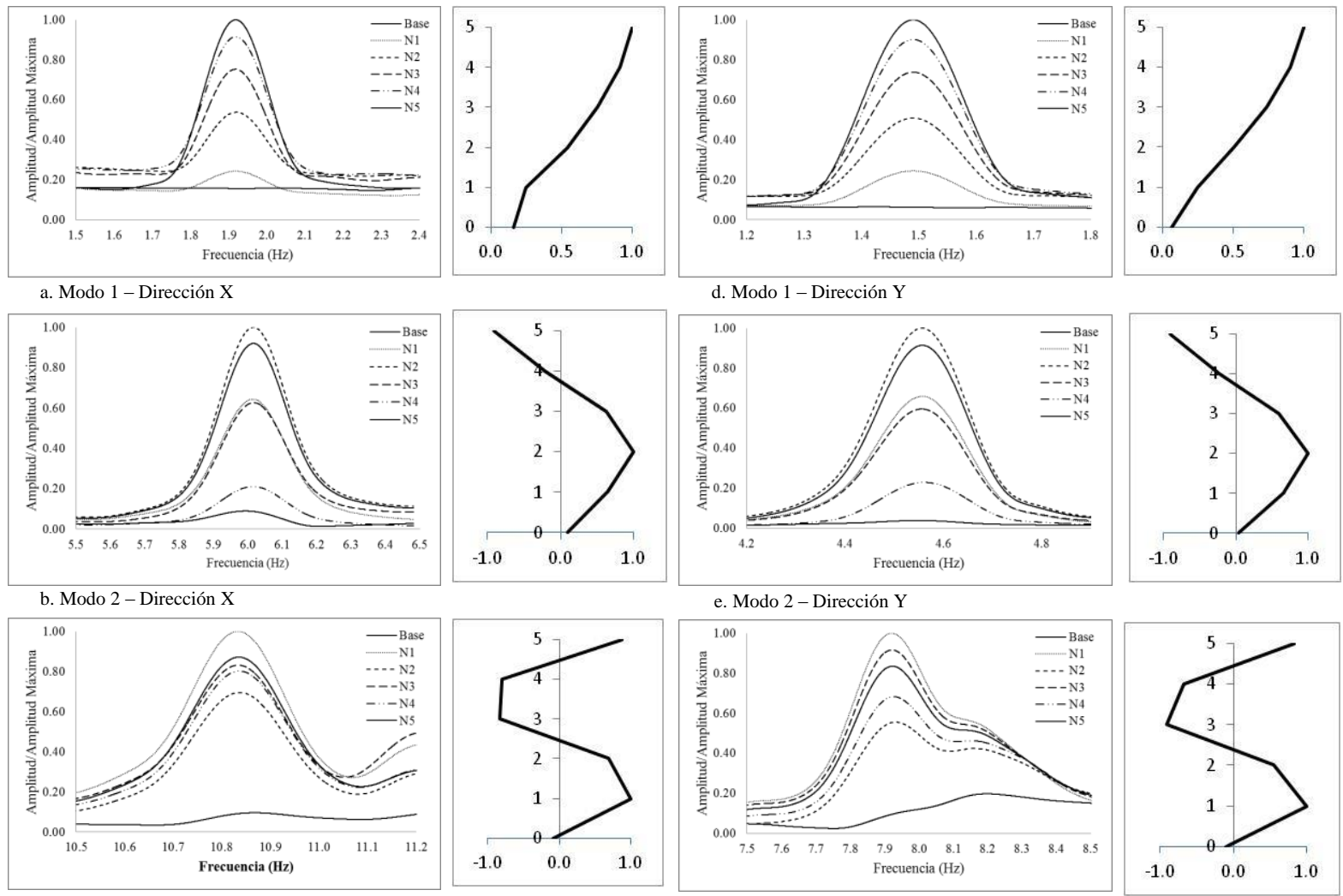

c. Modo 3 - Dirección X
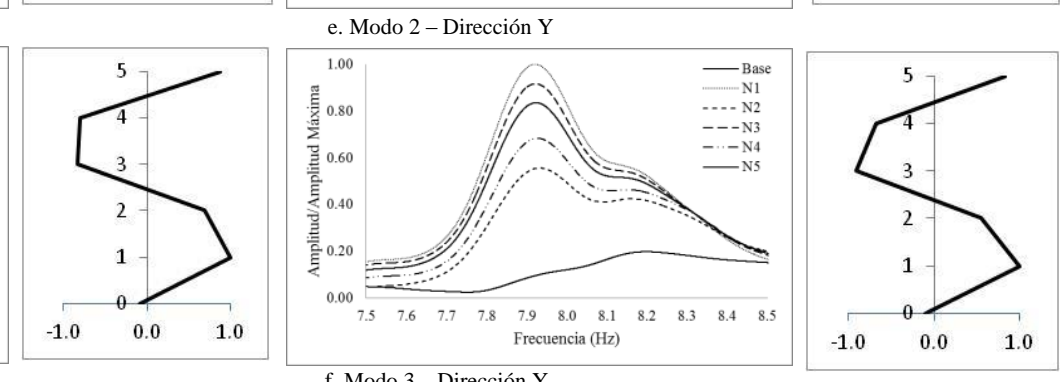

Figura 9. Formas modales de los tres primeros modos en cada dirección obtenidos para las pruebas de vibración ambiental en traslación

\section{Pruebas de vibración ambiental para determinar el amortiguamiento}

Se obtienen los espectros de potencia de la señal de la azotea calculados mediante la metodología de Welch (1967), para diferentes longitudes de ventanas o tramos secuenciales de los registros y se aplica la metodología del ancho de banda corregido (Boroschek y Hernandez, 2010). 


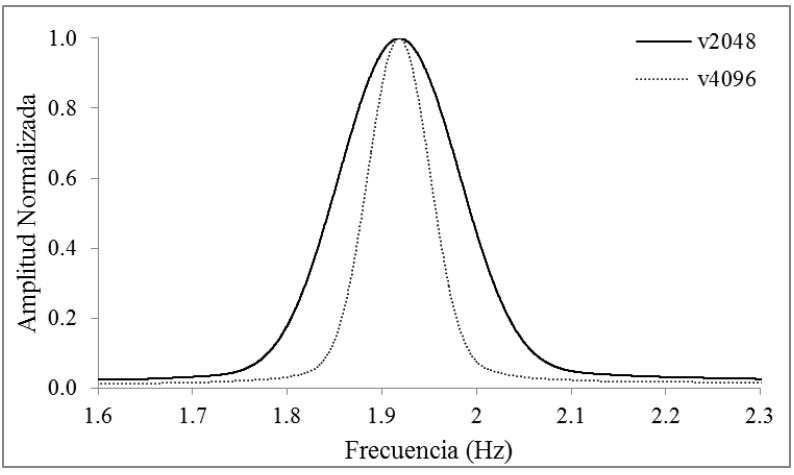

Figura 10. Espectro de potencia normalizado para cálculo del amortiguamiento

La Figura 10 muestra el espectro de potencia de la señal de la azotea, normalizado para el primer modo y calculado para dos longitudes de ventanas promediadas (2048 y 4096 puntos). Cabe resaltar como el ancho de banda disminuye a medida que se aumenta la longitud de las ventanas, por lo que se obtendrían valores diferentes de fracciones de amortiguamiento crítico si se empleara únicamente el método del ancho de banda de Kawasumi y Shima (1965). Por este motivo se aplica la corrección descrita por Boroschek y Hernandez (2010) para estimar un valor de la fracción de amortiguamiento crítico confiable.

La Tabla 3 muestra el amortiguamiento calculado para los tres primeros modos en las dos direcciones ortogonales y para las dos longitudes de ventanas mostrados. Se observa la diferencia que existe entre los amortiguamientos sin corrección y la consistencia que existe entre los valores calculados corregidos.

Tabla 3. Amortiguamiento calculado

\begin{tabular}{cccccc}
\hline Modo & $\boldsymbol{f ( H z )}$ & $\begin{array}{c}\boldsymbol{\xi}(\%) v 2048 \\
\text { Sin } \\
\text { Corrección }\end{array}$ & $\begin{array}{c}\boldsymbol{\xi}(\%) v 2048 \\
\text { Corregida }\end{array}$ & $\begin{array}{c}\xi(\%) \text { v4096 } \\
\text { Sin } \\
\text { Corrección }\end{array}$ & $\begin{array}{c}\xi(\%) v 4096 \\
\text { Corregida }\end{array}$ \\
\hline $1 \mathrm{x}$ & 1.9 & 3.9 & 0.4 & 2.0 & 0.3 \\
$1 \mathrm{y}$ & 1.5 & 4.9 & 0.3 & 2.5 & 0.3 \\
$2 \mathrm{x}$ & 6.1 & 1.4 & 0.5 & 0.9 & 0.5 \\
$2 \mathrm{y}$ & 4.6 & 1.7 & 0.3 & 1.0 & 0.4 \\
$3 \mathrm{x}$ & 10.8 & 0.8 & 0.3 & 0.6 & 0.4 \\
$3 \mathrm{y}$ & 7.9 & 1.4 & 0.8 & 0.9 & 0.7 \\
\hline
\end{tabular}

\section{Pruebas de vibración ambiental para identificar propiedades de torsión}

Para la identificación de las frecuencias de vibración asociadas a torsión, se utiliza el arreglo de sensores descrito para este fin, y se realizan comparaciones entre los puntos instrumentados en un mismo nivel del modelo. De esta manera, se plantean dos tipos de comparaciones para la identificación de las propiedades del sistema: una comparación entre el sensor ubicado en el centro y otro en uno de los extremos, y otra comparación entre los puntos extremos de la edificación instrumentados. 
En la Figura 11 se grafican los espectros de potencia de los registros de un mismo nivel, y a partir de éstos es posible abstraer primeros indicios acerca de las frecuencias de torsión. Se observa que en los puntos donde los gráficos de los sensores de los extremos exhiben picos que el del centro no muestra, se identifica una posible frecuencia de torsión. Es recomendable realizar el análisis espectral completo, pues al ser las amplitudes tan bajas puede haber picos no correspondientes a la información requerida.
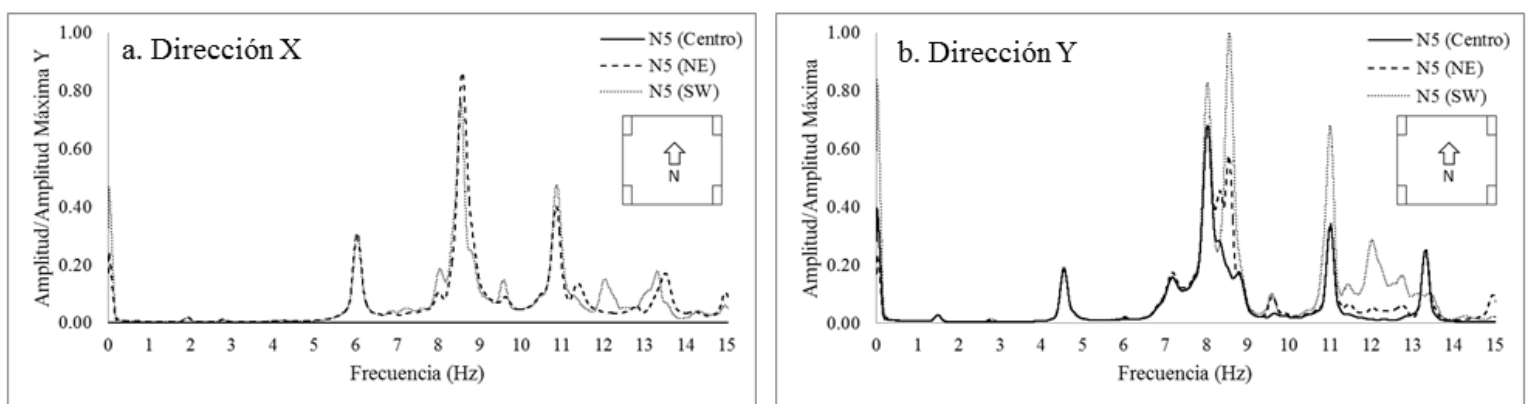

Figura 11. Espectros de potencia obtenidos a partir de los registros de los sensores ubicados en el nivel N5 (azotea) para las pruebas de vibración ambiental en torsión

La Figura 12 muestra un acercamiento a las frecuencias identificadas, donde se puede observar con mejor resolución la propiedad descrita.

Cuando se comparan los puntos instrumentados, uno en el centro y otro en el extremo de un mismo nivel, se analiza la información contenida en los cocientes espectrales, ya que los movimientos rotacionales asociados a torsión, se deben mostrar únicamente los registros de los sensores de los extremos, más no en el central. La Figura 13 muestra el cociente espectral entre los espectros de potencia de los registros de dos sensores ubicados uno en el extremo y otro en el centro del nivel 5; además de las frecuencias de torsión se muestran picos de amplitudes similares en otras frecuencias, por lo que este análisis por sí mismo no es plenamente concluyente, ya que no es posible aislarlos con claridad. Por esta razón este procedimiento se debe complementar con los demás análisis aquí descritos y revisar los resultados en conjunto.

Por otra parte, cuando se comparan los puntos de los extremos se analiza la información contenida en las funciones de coherencia y a la fase de los espectros cruzados. Estos puntos registran los movimientos rotacionales asociados a torsión pero en direcciones diferentes. 

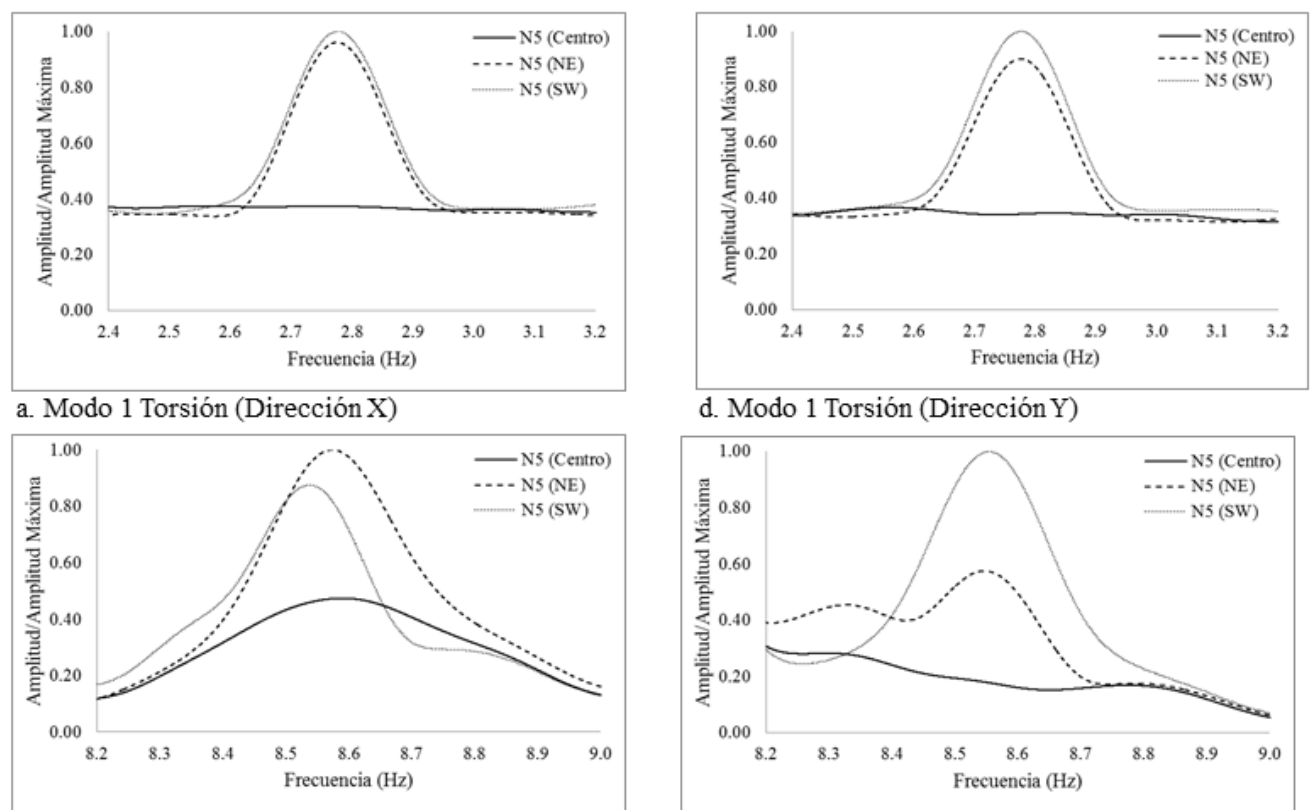

b. Modo 2 Torsión (Dirección X)

e. Modo 2 Torsión (Dirección Y)

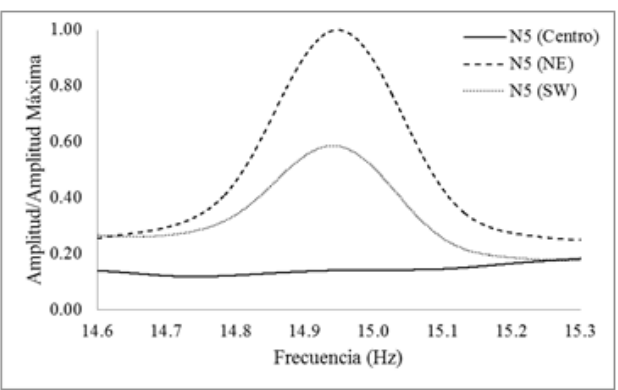

c. Modo 3 Torsión (Dirección X)

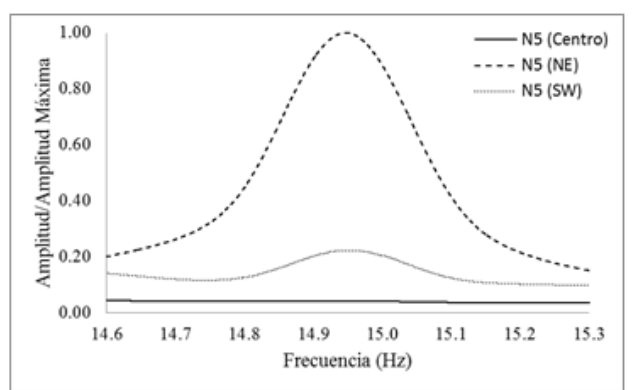

f. Modo 3 Torsión (Dirección Y)

Figura 12. Identificación de frecuencias de torsión obtenidas a partir de las pruebas de vibración ambiental

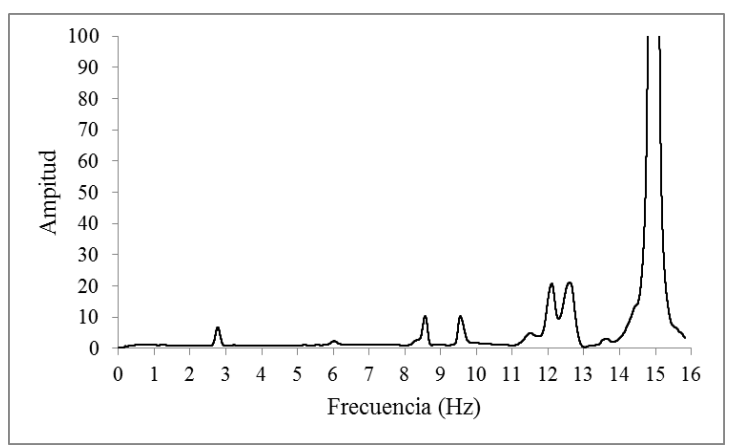

Figura 13. Cociente espectral entre los espectros de potencia obtenidos a partir de los registros de los sensores ubicados en el extremo y el centro del modelo para las pruebas de vibración ambiental en torsión 
La Figura 14 muestra la función de coherencia entre los puntos descritos, como ambos aparatos registran la señal de torsión, la función de coherencia es cercana a la unidad para estas frecuencias. Si bien el valor de esta función es alto para otras frecuencias, sirve de punto de validación para los resultados previamente identificados.

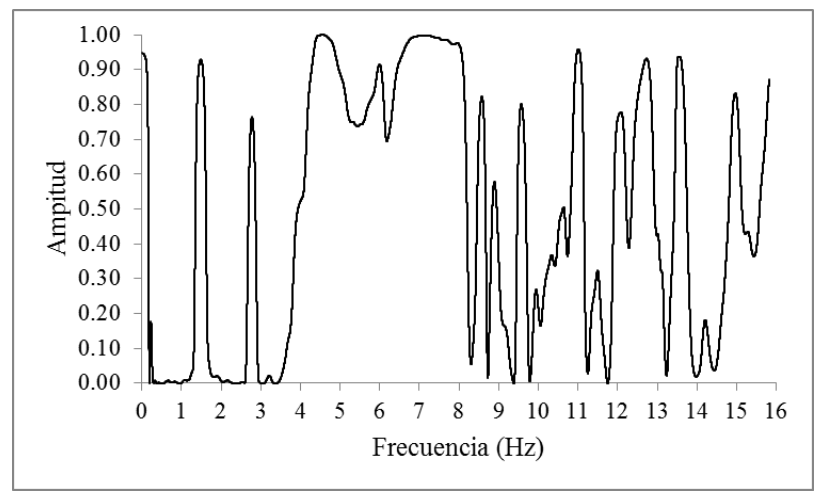

Figura 14. Función de coherencia obtenida a partir de los registros de los sensores ubicados en los extremos opuestos del modelo para las pruebas de vibración ambiental en torsión

La Figura 15 muestra el ángulo de fase entre las señales descritas, debido a la direccionalidad señalada, su valor en las frecuencias de torsión para estas comparaciones debe ser de $\pm \pi$.

Para el modelo estructural, con base en este análisis se identificaron las frecuencias de vibración de torsión en $2.7,8.5$ y $14.9 \mathrm{~Hz}$.

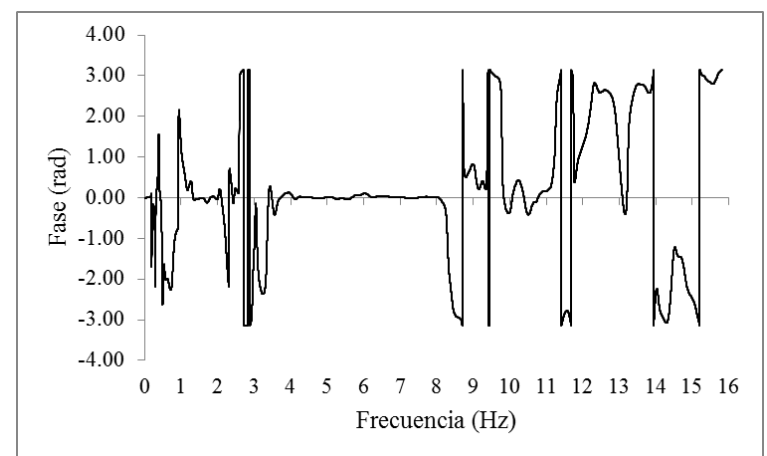

Figura 15. Espectro de ángulo de fase obtenido a partir de los registros de los sensores ubicados en los extremos opuestos del modelo para las pruebas de vibración ambiental en torsión 


\section{Pruebas de vibración forzada para identificar propiedades de traslación}

La Figura 16 muestra los registros obtenidos en la base y la azotea en las pruebas de vibración forzada. Cabe resaltar que la aceleración máxima en la base llega a $75 \mathrm{Gal}$, mientras que en la azotea alcanza casi $200 \mathrm{Gal}$.

Para este tipo de análisis de las señales que presentan este tipo de excitación, se utiliza el cociente entre los espectros de potencia de uno de los niveles de la estructura instrumentada (preferiblemente la azotea) y la base, el cual muestra picos en las frecuencias de traslación de la estructura.

La Figura 17 muestra el cociente descrito, en la cual se pueden observar picos en 1.88, 6.02 y 10.55 Hz. Estos valores se identifican como las frecuencias de traslación de la estructura.
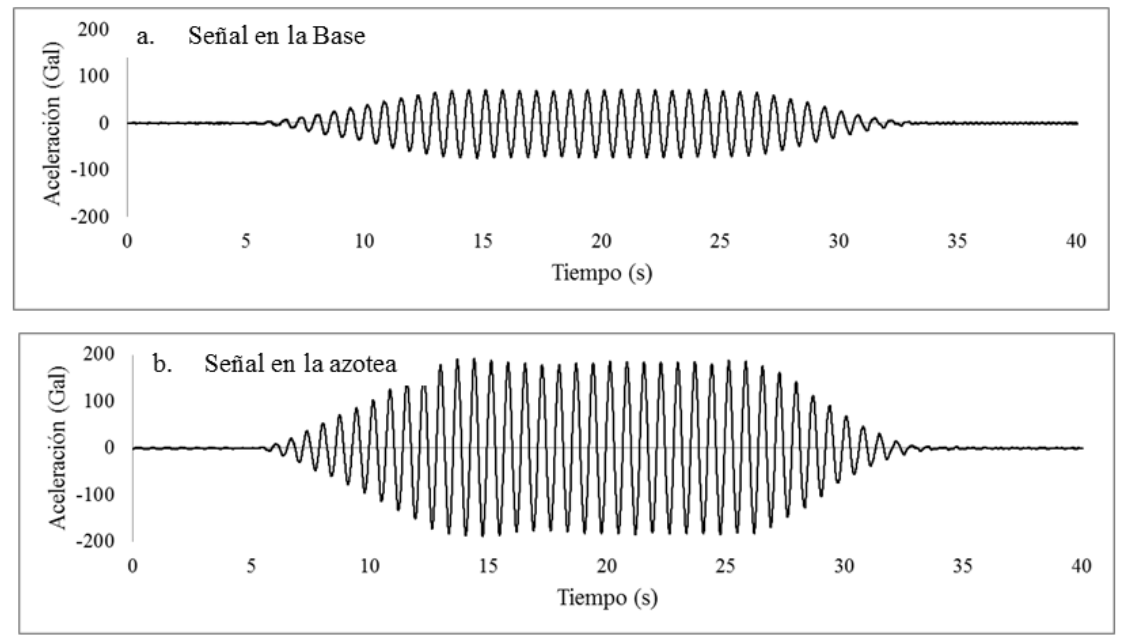

Figura 16. Señales en el tiempo obtenidas para las pruebas vibración forzada

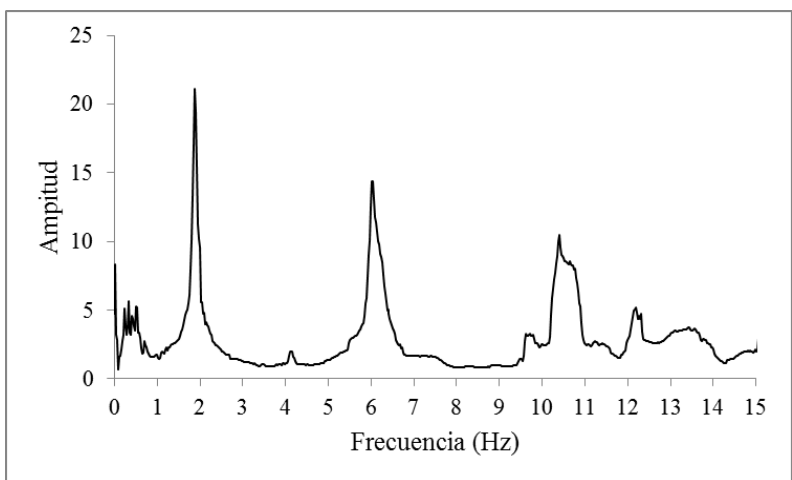

Figura 17. Cociente espectral entre los espectros de potencia obtenidos a partir de los registros de los sensores ubicados en la azotea y en la base para las pruebas de vibración forzada en traslación 


\section{Pruebas de vibración forzada para identificar propiedades de torsión}

Para el análisis de estas propiedades se hace igualmente uso de los cocientes espectrales, pero en este caso entre las señales de los sensores de los extremos con el del centro de un mismo nivel. Estos describen picos en los puntos asociados a las frecuencias de torsión.

La Figura 18 muestra el cociente espectral descrito para uno de los niveles instrumentados. Se identifican las frecuencias de torsión de los tres primeros modos en 2.7, 8.7 y $14.8 \mathrm{~Hz}$.

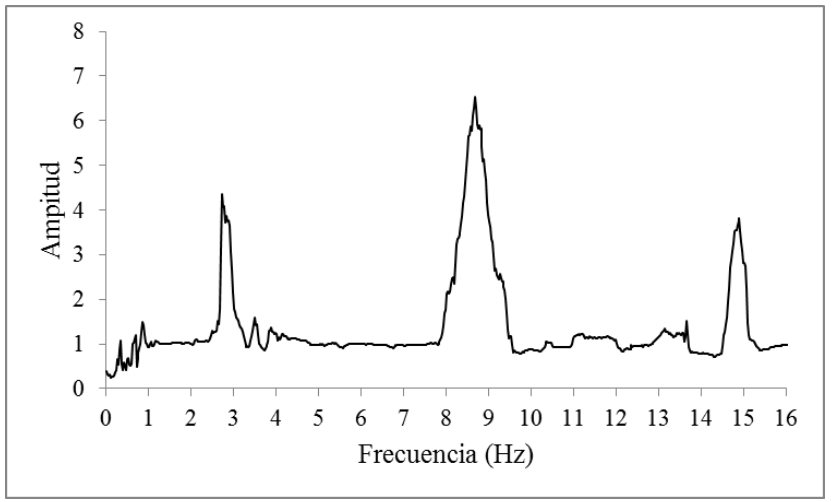

Figura 18. Cociente espectral entre los espectros de potencia obtenidos a partir de los registros de los sensores ubicados en los extremos opuestos de un mismo nivel para las pruebas de vibración forzada en torsión

Adicionalmente, como mecanismo de comprobación, es posible observar el espectro de fase entre las señales de los espectros de los extremos de un mismo nivel. Para las frecuencias identificadas como de torsión las señales deben estar en desfase, esto es, mostrar un valor de $\pm \pi$ (Figura 19).

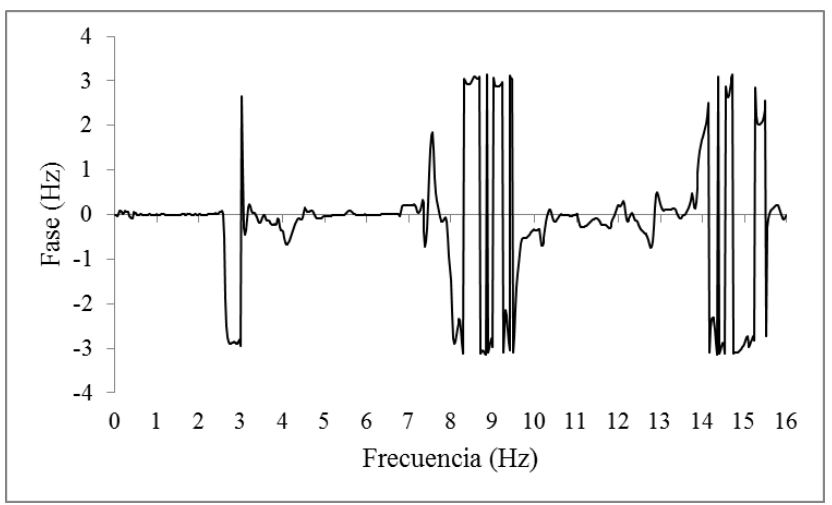

Figura 19. Espectro de ángulo de fase obtenido a partir de los registros de los sensores ubicados en los extremos opuestos de un mismo nivel del modelo para las pruebas de vibración forzada en torsión 


\section{COMPARACIÓN DE RESULTADOS}

La Tabla 4 muestra una comparación entre los resultados obtenidos en el modelo analítico y los resultados de los experimentos tanto para las pruebas de vibración ambiental como los de vibración forzada.

Para las frecuencias de vibración, en cuanto a los tipos de pruebas experimentales realizadas los resultados son muy similares entre sí. Además para los modelos analítico y experimental, los resultados obtenidos presentan igualmente pequeñas diferencias. Lo anterior permite validar la metodología empleada para el análisis e identificación de las frecuencias de vibración de una estructura sometida tanto a vibración ambiental como vibración forzada, toda vez que los valores obtenidos para los casos de análisis son consistentes con los valores de referencia y se evidencia una correlación alta para todos los datos obtenidos.

Tabla 4. Resultados analíticos y experimentales

\begin{tabular}{ccccccc}
\hline \multirow{2}{*}{ Modo } & \multicolumn{2}{c}{ Modelo Analítico } & \multicolumn{2}{c}{$\begin{array}{c}\text { Modelo Experimental } \\
\text { (Vibración Forzada) }\end{array}$} & \multicolumn{2}{c}{$\begin{array}{c}\text { Modelo Experimental } \\
\text { (Vibración Ambiental) }\end{array}$} \\
& $\boldsymbol{T}(\boldsymbol{s})$ & $\boldsymbol{f}(\mathrm{Hz})$ & $\boldsymbol{T}(\boldsymbol{s})$ & $\boldsymbol{f}(\boldsymbol{H z})$ & $\boldsymbol{T}(\boldsymbol{s})$ & $\boldsymbol{f}(\boldsymbol{H z})$ \\
\hline $1 \mathrm{x}$ & 0.51 & 1.9 & 0.53 & 1.9 & 0.53 & 1.9 \\
$1 \mathrm{y}$ & 0.66 & 1.5 & - & - & 0.67 & 1.5 \\
$1 \mathrm{z}$ & 0.37 & 2.7 & 0.37 & 2.7 & 0.36 & 2.7 \\
$2 \mathrm{x}$ & 0.17 & 6.0 & 0.17 & 6.0 & 0.17 & 6.1 \\
$2 \mathrm{y}$ & 0.22 & 4.5 & - & - & 0.22 & 4.6 \\
$2 \mathrm{z}$ & 0.12 & 8.1 & 0.11 & 8.7 & 0.12 & 8.5 \\
$3 \mathrm{x}$ & 0.10 & 10.5 & 0.09 & 10.5 & 0.09 & 10.8 \\
$3 \mathrm{y}$ & 0.14 & 7.3 & - & - & 0.13 & 7.9 \\
$3 \mathrm{z}$ & 0.07 & 13.6 & 0.07 & 14.8 & 0.07 & 14.9 \\
\hline
\end{tabular}

\section{CONCLUSIONES}

Los resultados obtenidos en la identificación de las propiedades dinámicas del modelo estructural de cinco niveles construido en acero, resultan ser muy similares cuando se comparan las diferentes pruebas experimentales de vibración ambiental y vibración forzada con el modelo analítico, lo que permite validar la adopción de la metodología espectral empleada. Asimismo, los resultados obtenidos a partir del análisis experimental de la estructura son confiables, toda vez que se validaron con resultados de modelos analíticos empleados en el diseño dinámico convencional de edificaciones. Esto implica que los equipos adquiridos recientemente por la Universidad EAFIT arrojan resultados coherentes y consistentes con las propiedades dinámicas de las estructuras analizadas.

La localización espacial de los sensores durante la instrumentación de una edificación se debe realizar dependiendo de los parámetros que se desean medir, con el fin de optimizar la cantidad de equipos de los que se dispone. De esta manera, si se desea medir frecuencias traslacionales es importante ubicar los instrumentos en diferentes niveles en altura y uno en la base. Por otra parte, si se desean medir frecuencias de torsión se debe disponer de un arreglo de al menos dos sensores en uno de los niveles de análisis (preferiblemente el último) con el fin de identificar los movimientos relativos de rotación. 
Para la adecuada identificación de las propiedades dinámicas de una estructura se requieren aparatos que registren en forma simultánea las vibraciones en diferentes puntos de la estructura. La información de un solo aparato puede ser útil pero no suficiente, ya que un correcto análisis de las frecuencias modales, es necesario realizar comparaciones entre los resultados de diferentes sensores. Adicionalmente, las amplitudes del movimiento de la estructura en vibraciones ambientales son tan bajas que los resultados pueden dar información incorrecta de otro tipo de movimiento o excitaciones diferentes a la estructura por lo que se requiere del análisis espectral completo.

Para la identificación de las frecuencias de traslación de una estructura en pruebas de vibración ambiental mediante el uso de la teoría de datos aleatorios, se reconocen dos tipos de análisis los cuales se deben realizar de manera conjunta: en el primero se comparan puntos instrumentados dentro de la estructura con puntos fuera de ésta; aquí la identificación de las frecuencias se hace mediante la identificación de picos en los cocientes espectrales. En el segundo tipo de análisis, se comparan puntos dentro de la misma estructura; éste sirve para confirmar las frecuencias identificadas con el primer análisis, mediante la función de coherencia principalmente. Esta metodología debe ir acompañada por el estudio de la información contenida en los espectros de potencia.

La identificación de las frecuencias asociadas a los modos de torsión se lleva a cabo mediante la instrumentación de un mismo nivel de la estructura en al menos dos puntos diferentes. Las frecuencias de rotación se observan principalmente al comparar los espectros de potencia de los puntos instrumentados. Los espectros de sensores ubicados cerca de los extremos de la estructura deberán presentar picos asociados a las frecuencias de rotación que deberán tener amplitudes significativamente más bajas en sensores cerca del centro, adicionalmente para sensores ubicados en extremos opuestos, las frecuencias de torsión deberán estar en desfase. También se utilizan cocientes espectrales y funciones de coherencia con un análisis análogo al utilizado para identificar las frecuencias de flexión.

El método del ancho de banda del espectro de potencia permite la identificación de la fracción de amortiguamiento crítico de cada modo de vibración de la estructura. Se concluye que para su aplicación es indispensable el empleo de un método de corrección por sobrestimación, ya que el mecanismo de cálculo numérico de los espectros de potencia con diferente número de ventanas, produce una sobre apreciación de éste parámetro. Se utilizó la formulación de corrección planteada por Boroschek y Hernandez (2010) obteniendo resultados consistentes.

\section{AGRADECIMIENTOS}

Los autores agradecen a la Universidad EAFIT por brindar los medios económicos para el desarrollo de la investigación cuyos resultados se presentan en este artículo.

\section{BIBLIOGRAFÍA}

Bendat, J. y Piersol, A. G., (1993). Engineering applications of correlation and spectral analysis. New York: John Wiley \& Sons.

Bendat, J. S. y Piersol, A. G., (2010). Random Data. Analysis and Measurement Procedures. 4ta ed. Hoboken(NJ): John Wiley \& Sons. 
Boroschek, R. y Hernandez, F., (2010). “Corrección de sobreestimación del amortiguamiento en el método de ancho de banda del espectro de potencia". Congreso chileno de sismología e ingeniería antisísmica.

Computers and Structures Inc., (2012). SAP2000 v15.0, Integrated Software for analysis and design. Berkeley: s.n.

Kawasumi, H. y Shima, E., (1965). "Some applications of a correlator to engineering problems". Proceedings on the third world conference on earthquake engineering, pp. II298-II320.

Murià Vila, D., (2007). "Experiencia Mexicana sobre la respuesta sísmica de edificios instrumentados". Mexico D.F.: Academia de Ingeniería, Mexico.

Murià Vila, D. y Gonzalez Alcorta, R., (1995). "Propiedades dinámicas de edificios de la ciudad de México". Revista de Ineniería Sísmica, n 51, pp. 25-45.

Trifunac, M. D., (1972). “Comparisons between ambient and forced vibration experiments". Earthquake engineering and structural dymanics, Volumen I, pp. 133-150.

Welch, P. D., (1967). "The use of fast fourier transform for the estimation of power spectra: A method based on time averaging over short, modified periodograms". IEEE Trans. Audio Electroacustics, Volumen AU-15, pp. 70-73. 